\title{
Increasing the efficiency of wind turbines
}

\author{
Asaf Varol ${ }^{\mathrm{a}, *}$, Cumali İlkılıç ${ }^{\mathrm{b}}$, Yasin Varol ${ }^{\mathrm{c}}$ \\ ${ }^{a}$ Communications Faculty, Firat University, 23119 Elazı̆̆, Turkey \\ ${ }^{\mathrm{b}}$ Technical Education Faculty, Department of Material Science, Firat University, 23119 Elazı̆̆, Turkey \\ ${ }^{\mathrm{c}}$ Technical Education Faculty, Department of Mechanical Science, Firat University, 23119 Elaziğ, Turkey
}

\begin{abstract}
The importance of clean energy sources was realised rapidly after the negative effects of the pollution caused by generators on the environment became clear. Wind energy is a clean and renewable energy source whose applications exist worldwide. Some countries changed their national energy forward planning for renewable clean energy projects. In developed countries some researchers have worked on developing new kinds of wind turbines in order to produce a part of their power from renewable wind energy. The rotational speed of the wind blades can be increased using steering aerofoils surrounding the blades. A prototype of this wind turbine has been made in the laboratory; a ventilator whose flow rate is variable generates the 'wind'. The blade profiles are designed using the theory of aerodynamics. The steering aerofoils are fixed surrounding the wind blades at an optimum distance. The number of the aerofoils and the angle of inclination (tilt) of the foils can be changed. In the experiment the ambient conditions are held constant. Because of the optimum adjustment of the distance and angle of the aerofoils the rotational speed of the blades can be increased by $32 \%$ on the experimental device. In this paper the theoretical and experimental results will be discussed. (C) 2001 Elsevier Science Ltd. All rights reserved.
\end{abstract}

Keywords: Wind Energy; Efficiency; Aerofoils

\section{Introduction}

Wind energy results from the different temperature layers of the atmosphere and the unevenness of the earth's surface. The low- and high-pressure areas occur in the atmosphere because of temperature differences. Due to the pressure differences, air flows from the high-pressure region to the low-pressure region, which of course is

*Corresponding author. 
wind. Wind energy can be harnessed directly at suitable locations unlike fossil fuels such as coal, which require transportation and treatment before processing.

There are several applications of wind energy particularly in generating electricity. This has been attempted by converting this energy into rotating energy using a wind turbine to drive the electrical generators. The advantages of this type of energy are cheap source and no damage to the environment.

In the past much research had been done in this field particularly from the point of view of velocity direction control of the wing and in turbine blade design. Most of the previous work has been concentrated on wind blades design.

Rechenberg [1] has carried out research on wing turbine with vortex screw concentrator. There is a need for detailed investigation into using variable steering aerofoils surrounding the wind blades.

\section{Wind blade theory}

From Euler Theorem, the force on the wind blade is

$$
F=r A_{\mathrm{T}} V_{\mathrm{T}}\left(V_{1}-V_{2}\right),
$$

where $V_{1}$ is the inlet wind velocity $(\mathrm{m} / \mathrm{s}), V_{2}$ the outlet wind velocity $(\mathrm{m} / \mathrm{s}), A_{\mathrm{T}}$ the swept wind area surrounding the wind blade $\left(\mathrm{m}^{2}\right), V_{\mathrm{T}}$ the average wind velocity, which is rotating the blades $(\mathrm{m} / \mathrm{s}), r$ the air density $\left(\mathrm{kg} / \mathrm{m}^{3}\right)$.

The power on wind blades axle is

$$
P=F V_{\mathrm{T}}=r A_{\mathrm{T}} V_{\mathrm{T}}^{2}\left(V_{1}-V_{2}\right) .
$$

The power, which comes from the wind energy, is proportional in third order of the wind velocity. If the wind velocity is doubled the power will be approximately increased eight times.

The kinetic power exchange of the wind before entering and leaving the wind blades is

$$
D_{\mathrm{EK}}=\frac{1}{2} m_{A}\left(V_{1}^{2}-V_{2}^{2}\right),
$$

$m_{A}$ in above equation is mass flow of the wind and equal to

$$
m_{A}=r A_{\mathrm{T}} V_{\mathrm{T}} \text {. }
$$

The power to drive the wind blades is equal to the kinetic power exchange assuming there are no losses; hence,

$$
\begin{aligned}
& P=D_{\mathrm{EK}}, \\
& r A_{\mathrm{T}} V_{\mathrm{T}}^{2}\left(V_{1}-V_{2}\right)=\frac{1}{2} r A_{\mathrm{T}} V_{\mathrm{T}}\left(V_{1}^{2}-V_{2}^{2}\right) .
\end{aligned}
$$

From the Eq. (6)

$$
V_{\mathrm{T}}=\left(V_{1}+V_{2}\right) / 2 \text {. }
$$

From the Eqs. (2) and (7) the total power equation is

$$
P=\frac{1}{4} r A_{\mathrm{T}}\left(V_{1}^{2}-V_{2}^{2}\right)\left(V_{1}+V_{2}\right) \text {. }
$$


From differentiation of Eq. (8) and assuming that wind velocity $V_{1}$ is equal to $V_{0}$ (the ambient wind velocity), the wind velocity $\left(V_{2}\right)$ for maximum power is obtained

$$
\mathrm{d} P / \mathrm{d} V_{2}=\frac{1}{4} r A_{\mathrm{T}}\left(V_{0}+V_{2}\right)\left(V_{0}-3 V_{2}\right) .
$$

Hence, the optimum velocity of wind is found as follows:

$$
V_{2}=V_{0} / 3 \text {. }
$$

If the Eq. (10) is substituted in Eq. (8), the maximum power is obtained

$$
P_{\max }=\frac{8}{27} r A_{\mathrm{T}} V_{0}^{3} \text {. }
$$

Dividing Eq. (11) by kinetic energy equation results [2]

$$
P_{\max } / D_{\mathrm{EK}}=0.59 \text {. }
$$

This result shows that only $59 \%$ of the existing wind energy can be converted into useful wind energy.

\section{Wind blade tangential force}

The average wind velocity $V_{\mathrm{T}}$ is found from Eqs. (7) and (10)

$$
V_{\mathrm{T}}=\frac{2}{3} V_{0} \text {. }
$$

$U$ in Fig. 1 is the tangential velocity of the wind blade and $U$ is vertical to $V_{\mathrm{T}}$ velocity.

The start-up force $(F)$, which is affected along the blade area, is given in Eq. (14)

$$
F=\left(\frac{1}{2}\right)_{r} f c_{\mathrm{a}} C^{2},
$$

where $f$ is the wind blade area $\left(\mathrm{m}^{2}\right), C$ the resultant velocity $(\mathrm{m} / \mathrm{s}), c_{\mathrm{a}}$ the start-up coefficient (-).

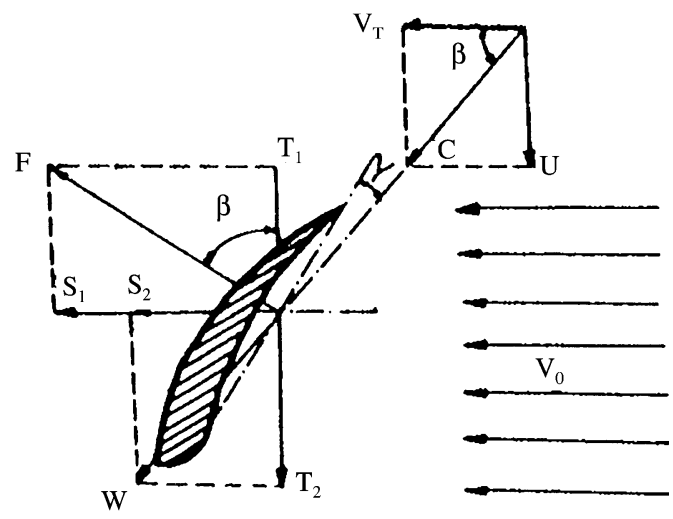

Fig. 1. The force distribution on the wind blade. 
$T 1$ is the component of the start-up force, which is vertical to the flow direction.

$$
T 1=F \operatorname{Cos} b \text {. }
$$

The resultant component in the flow direction is given as

$$
S=F \sin b+W \operatorname{Cos} b \gg F \sin b .
$$

The resistance force is neglected because of its lower value in comparison with start-up force.

If the length of the wind blade is $L$ and the width of the wind blade is $b$, then Eq. (14) results as

$$
F=0.5 r L b c_{\mathrm{a}} C^{2} \text {. }
$$

According to the wind blade theory, the force $(S)$ increases with the number of the wind blades $(z)$, therefore, total force in the flow direction is

$$
S=0.5 z r L b c_{\mathrm{a}} C U \text {. }
$$

This force is applied on the surface area of the flow $(2 p r L)$.

The flow volume is

$$
Q=A_{\mathrm{T}} V_{\mathrm{T}}=2 p r L V_{\mathrm{T}} .
$$

Substituting Eq. (19) in Eq. (1) results

$$
F=r Q\left(V_{1}-V_{2}\right) \text {. }
$$

The width of the wind blade is obtained from $S=F$ and using Eqs. (10), (13) and (19) and assuming that $V_{0}=V_{1}$

$$
b=(2 p r / z) \frac{8}{9}\left(c_{\mathrm{a}}\right)\left(V_{1}^{2} / U C\right) .
$$

\section{The wind blade and the steering aerofoils}

To increase the flow rate to the wind blade there is a need to increase the working area of the wind energy. The use of different auxiliary mechanisms could make this possible. Applying steering aerofoils in front of the wind blades causes a vortex, which improves and increases the flow area to the wind blades [1].

The effect of a vortex can be compared to the electrical wire coil, which causes electrical current flow in a magnetic field. Fig. 2 represents an analogy between electrical magnetic field and the vortex effect in aerofoil system [3].

In an electrical coil system, the increase in current results in an increase in the magnetic field $(H)$. In our analogy to the vortex case with the electrical system, the ambient velocity $V$ leads to an increase in the number of circulation vortices created. The number of vortices is related to the number of the aerofoils, which has an optimum number to avoid any turbulent problems. The creation of many vortices leads to a helical screw type motion as in Fig. 3. 

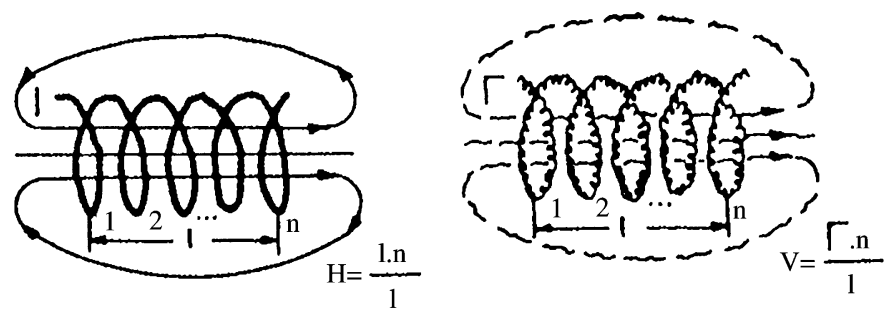

Fig. 2. Analogy between electrical magnetic field and the vortex effect in aerofoils system. $H=$ magnetic field, $I=$ current,$V=$ wind velocity, $G=$ Circulation, $l=$ length of coil or circulation, $n=$ number of turns of the coil or of the complete vortices.

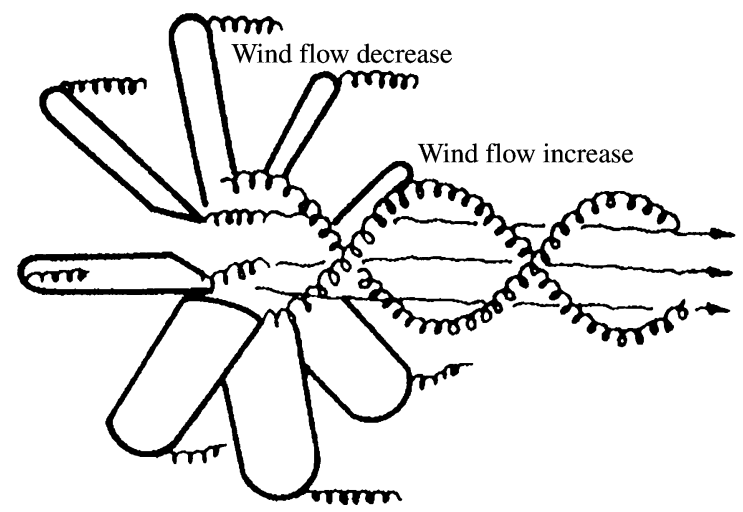

Fig. 3. Helical screw type motion.

The size of the vortices is identical with the same amplitude. The vortices with this helical motion tend to interact with each other and increase the flow velocity to the wind blade [3].

Due to the geometry and shape of the aerofoil there will be two helical screw type vortices, one initiating at the tip of the aerofoil. The outer vortex generated at the tip of the aerofoil decreases the wind flow while the inner vortex generated at the base of the aerofoil increases the wind flow. This is because the aerofoil increases the wind flow. This is due to the difference in the direction of rotation of the helical screw type motion of the vortices. The flow direction of both vortices is the same (Fig. 3). The flow velocity to the wind blade is controlled by the variation of the aerofoils angle [1].

\section{Experimental work and results}

The aerofoils were designed with the dimensions shown in Fig. 4. A set of the wind turbine was designed and constructed at the laboratory in order to study the effects of the aerofoils. 


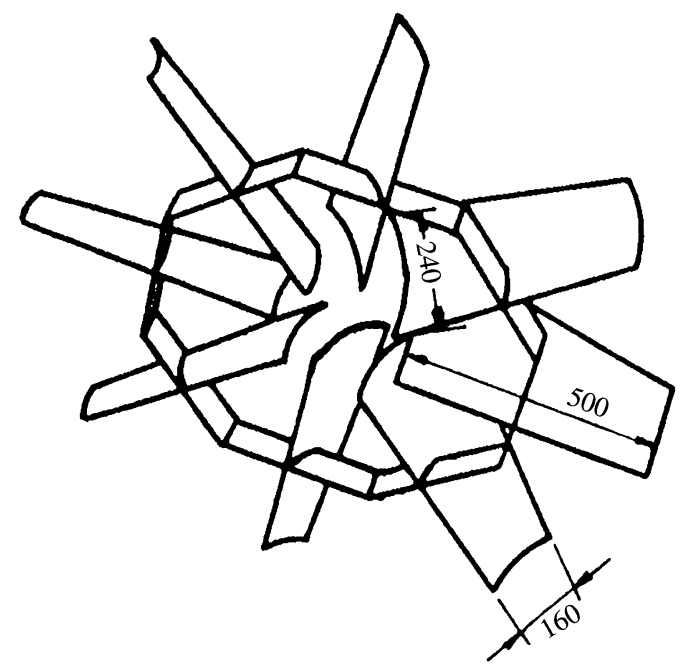

Fig. 4. Schematical view of the aerofoils.

The top tip (edge) of the aerofoils was $160 \mathrm{~mm}$ while the bottom edge was $240 \mathrm{~mm}$ and the length $500 \mathrm{~mm}$. The aerofoils were constructed using $2 \mathrm{~mm}$ nominal thickness steel. The aerofoils were fixed on an octagonal fan and their angles could be changed.

The wind was created using an axial ventilator. The ambient temperature was held constant during the experimental period. Changing the rotation speed of the ventilator can change the resulting wind velocity. The wind speed is measured using anemometers.

When the wind velocity is very high it can be decreased by adjusting the angle of the aerofoils. It is possible in this way to decrease the axial force at least 100 times. The rotation speed of the wind blade's rotor can be held constant by changing the angle of the aerofoils.

When the wind speed typically is $6.55 \mathrm{~m} / \mathrm{s}$ the rotation speed of the wind blades is $287 \mathrm{~min}^{-1}$ without using the aerofoils. If the experiments continue with aerofoils by a $6.55 \mathrm{~m} / \mathrm{s}$ wind speed the rotation speed can be increased to a value of $382 \mathrm{~min}^{-1}$. It means the $94 \mathrm{~min}^{-1}$ rotation speed is created using the aerofoils, which surround the wind blades, and the increase in the rotation speed is equal to $32 \%$.

The power produced at the axle of the wind blades is increased in the form of rotation speed and the moment generated. The higher the wind velocity the higher the rotation speed. The angle of the aerofoils can be changed whilst maintaining the other values constant.

In Fig. 5 the relationship between the rotation speeds of the wind blade axle and wind velocity is given when using aerofoils and without aerofoils on the rotation speed of wind blades especially at higher wind speeds. But is should be mentioned that if the angles of the aerofoils are not optimum it can effect the rotation speed negatively. 


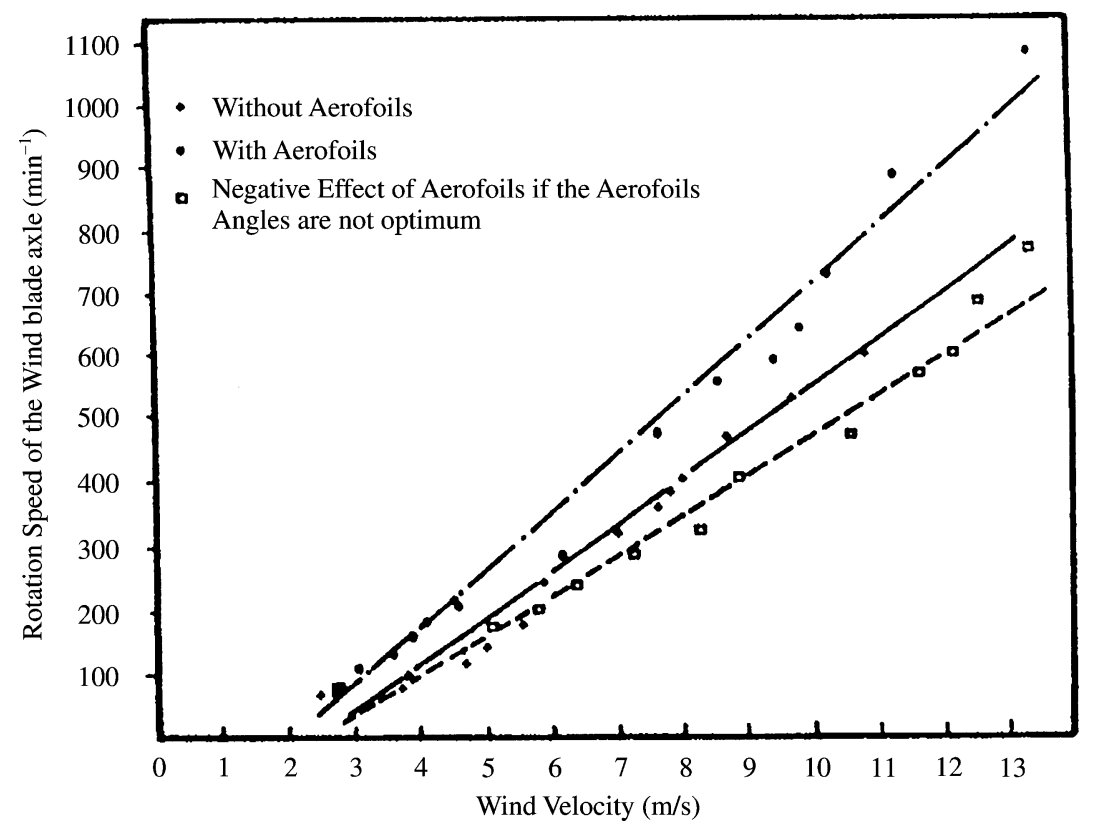

Fig. 5. The relationship between wind velocity and the rotation speed of the wind blade axle.

\section{Conclusion}

The research activities on wind energy seem to increase with time worldwide because wind energy is clean. It is important to increase the efficiency of the system.

The goal of this study was to show the positive effect of the aerofoils surrounding the wind blades. The idea of the surrounding aerofoils is similar to the steering blades of a water turbine like Francis or Kaplan turbine.

The direction of the wind in nature is changeable. Therefore the correct position of the aerofoils is very important to increase the rotation speed. If this is not in the correct position the aerofoils will affect the system negatively, which is shown in Fig. 5. The theoretical aspects of the relation between the aerofoils and wind blades are not investigated here. It is hoped that work along this direction will be continued.

\section{References}

[1] I. Rechenberg, Development and Operation of a novel Wind Turbine with Vortex Screw Concentrator, Second Joint Schlesinger Seminar on Energy and Environment, Berlin, 1989, pp. 1-11.

[2] T.F. Douglas, J.M. Gasiorek, J.A. Swaffield, Fluid Mechanics, Longman Scientific Technical, New York, 1985, pp. 317-328.

[3] I. Rechenberg, Berwian konzentriert den wind, Sonnen Energ. 4 (1984) 6-10. 